02

\title{
Влияние неравновесных условий синтеза на структуру и оптические свойства аморфных углеродных пленок
}

\author{
(C) А.П. Рягузов ${ }^{1}$, Р.Р. Немкаева ${ }^{1,2}$, О.И. Юхновец ${ }^{1}$, Н.Р. Гусейнов ${ }^{1}$, С.Л. Михайлова ${ }^{3}$, Ф. Бекмурат ${ }^{3}$, \\ А.Р. Асембаева ${ }^{1,4}$ \\ ${ }^{1}$ Национальная нанотехнологическая лаборатория открытого типа, \\ Казахский национальный университет им. аль-Фараби, \\ 050040 Алматы, Казахстан \\ 2 Лаборатория инженерного профиля, Казахский национальный университет им. аль-Фараби, \\ 050040 Алматы, Казахстан \\ ${ }^{3}$ Кафедра фризики твердого тела и нелинейной фризики, Казахский национальный университет им. аль-Фараби, \\ 050040 Алматы, Казахстан \\ ${ }^{4}$ Казахский национальный технический университет им. Сатпаева, \\ 050040 Алматы, Казахстан \\ e-mail: ryaguzov_a@mail.ru
}

Поступила в редакцию 29.01.2019 г.

В окончательной редакции 29.01.2019 г.

Принята к публикации 09.04.2019 г.

Аморфные углеродные (a-C) тонкие пленки синтезированы неравновесным методом ионно-плазменного распыления графитовой мишени в атмосфере аргона при постоянном значении тока плазмы. Методом рамановской спектроскопии проведены исследования локальной структуры углеродных пленок. Используя нормальное распределение в разложении рамановских спектров, выявили, что при температурах синтеза менее $150^{\circ} \mathrm{C}$ появляется пик в области частоты $1260 \mathrm{~cm}^{-1}$, характерный для фононной плотности состояний c $s p^{3}$-гибридизацией связей. Кроме этого, показано влияние размеров наноструктур из $s p^{2}$-узлов на оптическую ширину запрещенной зоны и их корреляцию с результатами рентгеновской фотоэлектронной спектроскопии (XPS) в а-C-пленках.

Ключевые слова: углеродные пленки, синтез, оптические свойства.

DOI: $10.21883 /$ OS.2019.08.48037.33-19

\section{Введение}

Существуют различные аллотропные формы углерода как в кристаллическом, так и в неупорядоченном состояниях [1]. Разнообразие аллотропных форм углерода определяется пространственным расположением $s p^{1}-, s p^{2}-$ и $s p^{3}$-гибридизированных связей. Степень гибридизации и ориентации химической связи влияет на распределение плотности энергетических состояний $\pi$ - и $\sigma$-электронов, что и является причиной отличия электронных свойств вещества.

Последние три десятилетия особое внимание уделяется изучению тонких аморфных алмазоподобных углеродных (DLC) пленок [2-7]. Существует много способов синтеза DLC-пленок в качестве покрытий, обладающих лучшими антифрикционными, прочностными и химически инертными свойствами [8-10]. Условия конденсации атомов определяют формирование атомной структуры пленок. В отличие от многих других методов синтеза магнетронный ионно-плазменный метод позволяет в широких пределах кинетических и термодинамических параметров синтеза выращивать аморфные DLC-пленки с заданной структурной модификацией. Неравновесные условия конденсации атомов углерода позволяют создавать различные кластерные структуры из $s p^{2}-$ и $s p^{3}$-узлов в аморфной углеродной матрице, и, таким образом, появляется возможность формировать наногетероморфные углеродные пленки с разными электронными свойствами.

Для корректировки условий синтеза и стабилизации процесса роста а-С-пленок использовали магнетрон с электромагнитом. Использование электромагнита позволяет регулировать плотность силовых линий магнитного поля на поверхности мишени, и это, как следствие, дает возможность корректировать ток плазмы и напряжение на катоде. Появление возможности контроля состояния плазмы позволяет фиксировать процесс распыления и конденсации и формирование структуры углеродных пленок в процессе синтеза. Таким образом, появляется возможность получать аморфные DLC-пленки с заданной структурной конфигурацией из $s p^{2}-$ и $s p^{3}$-узлов.

Для оценки соотношения $s p^{2} / s p^{3}$-узлов и выявления размеров кластерных образований из $s p^{2}$-узлов использовали рамановскую спектроскопию (RS). RS можно отнести к относительно щадящим методам исследования локальной атомной структуры. Используя накопленный материал спектральных данных по RS, можно проводить оценку процентного соотношения $s p^{2} / s p^{3}$-связей как, например, в работах [11-14]. В рамановских спектрах a-C-пленок полуширина основного $G$-пика, отношение 
интенсивностей пиков $I(D) / I(G)$, положение $G$-пика и его дисперсия [12] характеризуются вибрационными модами молекулярных групп из $s p^{2}$-узлов. Это связано с сечением рассеяния $s p^{2}$-узлов в видимом диапазоне возбуждающего излучения, которое в 50-250 раз больше, чем у $s p^{3}$-узлов, и характеризуется резонансными переходами между $\pi-\pi^{*}$-состояниями $[12,15,16]$. Энергия видимого излучения гораздо меньше, чем возбуждение более глубоко лежащих $\sigma$-состояний. Тем не менее, $\mathrm{RS}$-исследования структуры аморфных алмазоподобных углеродных пленок могут быть полезны в определении формирования $s p^{3}$-узлов в DLC-пленках.

Одним из важных критериев оценки результатов $\mathrm{RS}$ является оптическая ширина запрещенной зоны. Рассчитанная из спектров пропускания и отражения оптическая ширина запрещенной зоны относится к структурно-чувствительным параметрам, и поэтому может являться определенной оценкой соотношения $s p^{2} / s p^{3}$-гибридизированных связей $[1,12]$. Это связано с отличием в энергетическом состоянии $\pi$ и $\sigma$-электронов, формирующих зонную структуру. Энергетические состояния $\pi$ - и $\sigma$-электронов определяются конфигурацией связей между ближайшими соседями $s p^{2}$ - и $s p^{3}$-узлов [17].

Проведены исследования влияния температуры подложки, давления газа и удельной мощности разряда на структуру и оптические свойства. Результаты исследований коррелируют со значениями соотношения $s p^{2} / s p^{3}$-узлов, определенными из спектров XPS.

Выяснение влияния условий синтеза на формирование атомной структуры и соотношение $s p^{2} / s p^{3}$-узлов является основной задачей в исследовании электронных свойств наногетероморфных углеродных сред. Особенно это важно для формирования тонких структур в нанотехнологических процессах. Знание зависимости структуры и свойств от условий синтеза a-C-пленок позволит расширить понимание природы их формирования и возможности управления электронными процессами в новых устройствах наноэлектроники.

\section{Описание эксперимента}

Для синтеза углеродных пленок был использован охлаждаемый магнетрон с несбалансированной плазмой и регулируемой по величине индукцией (B) магнитного поля. Подаваемое напряжение $(U)$ от источника постоянного тока и электрон-ионный ток $(I)$ в плазме разряда дополнительно корректировались изменением В в процессе синтеза пленок. В менялась в пределах 25-40 mT. Устойчивость ионно-плазменного разряда и стабильность процесса плазменного распыления определяются отношением $E / p$, где $E-$ напряженность электрического поля между анодом и катодом магнетрона, $p$ - давление газа $\mathrm{Ar}$ в камере синтеза пленок. Корректировка индукции В магнитного поля позволяет при определенных условиях синтеза ( $U$ и $I$ разряда) поддерживать $E / p=$ const. Благодаря постоянству значения отношения $E / p$ в процессе синтеза стало возможно детальное изучение влияния термодинамических и кинетических параметров на структуру и оптические свойства синтезируемых аморфных углеродных пленок. Все образцы в работе были получены при одном значении силы тока (DS) в плазме.

При синтезе пленок удельная мощность $\left(P_{\mathrm{d}}\right)$ ионноплазменного разряда принимала значения 2.1, 2.4 и $2.6 \mathrm{~W} / \mathrm{cm}^{2}$. Рост углеродных пленок осуществлялся при трех температурах $\left(T_{\text {sub }}\right) 50,150$ и $250^{\circ} \mathrm{C}$ и давлениях газа аргон в интервале 0.5-1.2 Ра на кварцевых и кремниевых подложках. Чистота газа аргона и графитовой мишени составляло 99.999 at.\%. Время синтеза всех а-С-пленок было постоянно и равнялось $40 \mathrm{~min}$ со средней скоростью роста $\sim 15 \AA / \mathrm{min}$. Толщина пленок определялась на грани свежего скола кремниевой пластины в растровом электронном микроскопе Quanta 200i 3D и изменялась от 50 до $70 \mathrm{~nm}$ в зависимости от условий синтеза. Конденсация атомов углерода на подложку осуществлялась в интервале углов от 15 до $30^{\circ} \mathrm{C}$ между нормалью к поверхности подложки и направлением их движения. Данные условия синтеза мало изучены и представляют определенный интерес в технологии получения тонкопленочных структур в наноэлектронике.

Исследования локальной структуры аморфных углеродных пленок проводились методом RS на установке NTegraSpectra (NT-MDT, Россия). В методе RS использовался твердотельный лазер с длиной волны $473 \mathrm{~nm}$ $(2.62 \mathrm{eV})$. Диаметр пятна на образце возбуждающего лазерного излучения был равен $2 \mu \mathrm{m}$, что определяло достаточно большую площадь облучения на поверхности пленки. Точность определения частот излучений фононов $\pm 4 \mathrm{~cm}^{-1}$. Время экспозиции измерений спектров составляла $30 \mathrm{~s}$. Кроме этого, во избежание воздействия лазерного излучения мощностью $1.5 \mathrm{~mW}$ на атомную структуру пленок экспонирование осуществлялось в движении со скоростью $\sim 10 \mu \mathrm{m} / \mathrm{s}$.

Оптическую ширину запрещенной зоны рассчитывали из спектров пропускания и отражения, полученных на приборе UV3600 (Shimadzu, Япония). Все измерения структуры и оптических свойств были выполнены на свежеприготовленных образцах.

Измерения XPS проводились на установке KRATOS AXIS 165 XPS (в Миссурийском университете науки и технологии). Спектры регистрировали электронный поток с поверхностного слоя пленки толщиной $10 \mathrm{~nm}$. Измерения проводились в вакууме $\left(2 \cdot 10^{-8}\right.$ Torr) с использованием монохроматического излучения $\mathrm{Al} K_{\alpha}=1486.71 \mathrm{eV}$. Инструментальная погрешность измерения составляла $\pm 0.05 \mathrm{eV}$. Важно отметить, что при измерениях пленок на кремниевых подложках происходила зарядка поверхности. Поэтому при обработке данных учитывалось „зарядовое смещение“. 


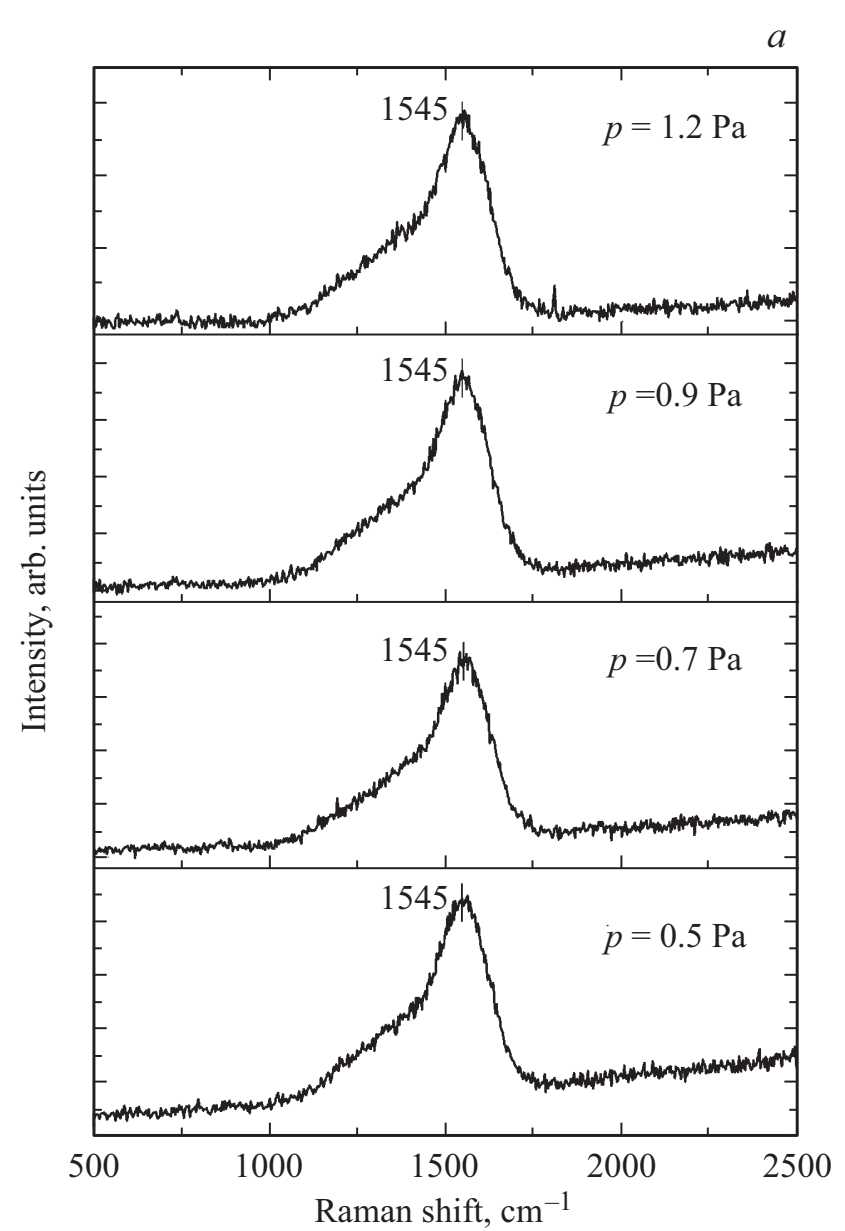

Рис. 1. Рамановские спектры а-С-пленок синтезированных при DC-разряда $2.1 \mathrm{~W} / \mathrm{cm}^{2}$ на кварцевых подложках.

\section{Результаты и обсуждение}

\section{Рамановская спектроскопия}

Согласно работам [1,12-16], рамановские спектры углеродных пленок с неупорядоченной структурой могут быть охарактеризованы двумя резкими максимумами, колебательные моды которых обозначаются как $D$-пик (Disordered) и $G$-пик (Graphitic) в областях частот $1350-1400 \mathrm{~cm}^{-1}$ и $1575-1600 \mathrm{~cm}^{-1}$ соответственно. Изменение положения $D$ - и $G$-пиков, отношение их интенсивностей определяют уровень гибридизации $\mathrm{C}-\mathrm{C}$ связей и изменение аллотропических форм в синтезируемых а-С-пленках от графитоподобной до алмазоподобной структуры.

Как известно, появление $G$-пика обусловлено модой центральной зоны $E_{2 g}$-симметрии растягивающих колебаний гексагональных углеродных колец [1]. В то же время присутствие $G$-пика в рамановских спектрах аморфных углеродных пленок с неупорядоченной структурой может быть связано с модами растяжения пар атомов $\mathrm{C}-\mathrm{C} s p^{2}$-узлов. Отсутствие шестигранных молекул $\mathrm{C}_{6}$ не исключает существование пар атомов

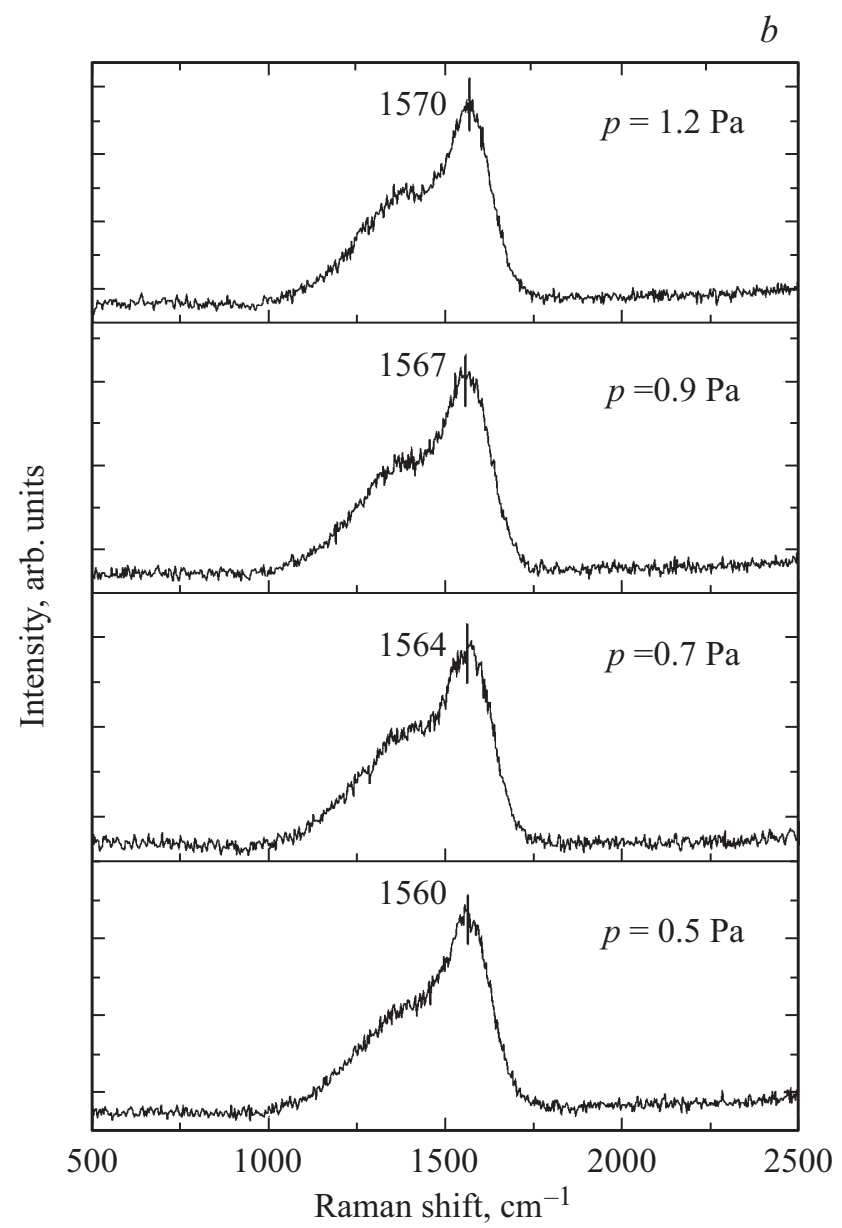
(a) $T_{\mathrm{sub}}=50^{\circ} \mathrm{C},\left(\right.$ b) $T_{\mathrm{sub}}=250^{\circ} \mathrm{C}$ и удельной мощности 


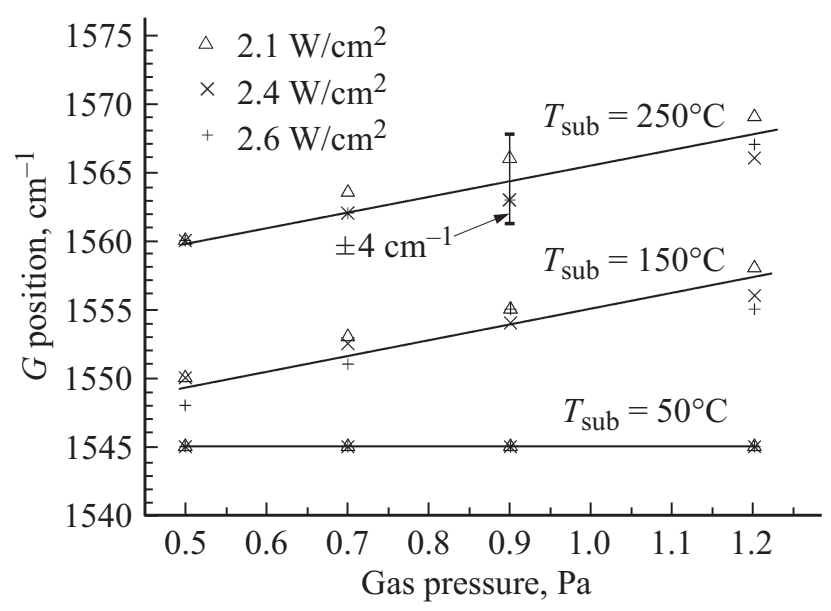

Рис. 2. Положение $G$-пика в зависимости от условия синтеза аморфных углеродных пленок на кварцевых подложках.

представленных на рис. $1, b$, при $T_{\text {sub }}=250^{\circ} \mathrm{C}$ положение $G$-пика существенно изменяет свое положение с увеличением давления и при $p_{\mathrm{Ar}}=1.2$ Ра принимает значение $1570 \mathrm{~cm}^{-1}$. Кроме этого, наблюдаем увеличение $D$-полосы в области $1360 \mathrm{~cm}^{-1}$. Смещение в высокочастотную область $G$-пика при $T_{\text {sub }}=250^{\circ} \mathrm{C}$ и увеличение интенсивности $D$-полосы свидетельствуют об увеличении $s p^{2} \mathrm{C}-\mathrm{C}-$ узлов и шестигранных колец $\mathrm{C}_{6}$.

Зависимость положения $G$-пика (PosG) от условий синтеза ( $T_{\text {sub }}$, давление газа $p$ и удельной мощности плазменного разряда $P_{\mathrm{d}}$ ) показана на рис. 2. Можно заметить, что функции $\operatorname{Pos} \mathrm{G}(p)$ для 150 и $250^{\circ} \mathrm{C}$ представляют собой почти параллельные линии с небольшим отклонением $\sim 4 \mathrm{~cm}^{-1}$ в зависимости от значений $P_{\mathrm{d}}$. Таким образом, можно предположить, что при данных температурах давление газа влияет на формирование структуры одинаковым образом. Однако при $T_{\text {sub }}=50^{\circ} \mathrm{C}$ положение $G$-пика не зависит от параметров синтеза $\left(p\right.$ и $\left.P_{\mathrm{d}}\right)$ и остается постоянным при $1545 \mathrm{~cm}^{-1}$.

Ранее мы исследовали влияние температуры подложки на структурные свойства а-С-пленок при других условиях синтеза и наблюдали положение $G$-пика при $1555 \mathrm{~cm}^{-1}$ для температуры $T_{\text {sub }}=50^{\circ} \mathrm{C}$ [11]. Таким образом, следует отметить, что стабилизация отношения $E / p$ в процессе синтеза позволяет получать а-С пленки с большим процентным содержанием $s p^{3}$-гибридизированных связей, так как смещение $G$-пика в низкочастотную область указывает на увеличение содержания $s p^{3}$-узлов. Как следует из работы [16], углеродные пленки с пиком $G$ при $1545 \mathrm{~cm}^{-1}$ более чем на $60 \%$ состоят из $s p^{3}$-узлов.

Авторы работы [18], используя метод синтеза а-Cпленок с фильтрацией ионов катодной дуги в вакууме (FCVA), обнаружили, что существует определенная активационная температура $T_{1}$, при которой происходит резкое изменение процесса формирования ближнего порядка атомной структуры. При этом было установлено, что процентное содержание $s p^{3}$ резко уменьшается при температуре синтеза $>200^{\circ} \mathrm{C}$. Кроме того, показано, что значение $T_{1}$ зависит от энергии конденсируемых атомов.

В нашей работе увеличение температуры синтеза приводит к изменению положения $G$-пика и его зависимости от давления газа при $T_{\text {sub }} 150$ и $250^{\circ} \mathrm{C}$. Следовательно, существует температура синтеза $T_{1}$ между 50 и $150^{\circ} \mathrm{C}$, при которой происходит переключение характера формирования структуры и ее зависимости от дополнительных кинетических и термодинамических параметров. Как видно из рис. $1, a$ и 2 , при $T_{\text {sub }}=50^{\circ} \mathrm{C}$ положение $G$-пика сохраняется при $1545 \mathrm{~cm}^{-1}$ и не зависит от мощности разряда и давления газа. Кроме того, важно отметить, что изменение удельной мощности ионноплазменного разряда (в пределах от 2.1 до $2.6 \mathrm{~W} / \mathrm{cm}^{2}$ ) при $T_{\text {sub }}=50^{\circ} \mathrm{C}$ не оказывает значительного влияния на формирование ближнего порядка структуры. Увеличение температуры синтеза приводит к смещению $G$-пика, и его положение начинает зависеть от давления газа (рис. $1, b$ и 2). Как видно из полученных результатов, изменение температуры подложки на $200^{\circ} \mathrm{C}$ приводит к смещению положения пика на $20 \pm 5 \mathrm{~cm}^{-1}$, что определяет существенное изменение соотношения $s p^{2} / s p^{3}$ и соответственно изменение в формировании структуры.

Для более детального понимания влияния условий синтеза на структуру аморфных углеродных пленок провели разложение спектров КРС на составляющие по методу Гаусса. Разложение по методу Гаусса представленных на рис. 3 спектров комбинационного рассеяния света характерно для всех аморфных углеродных пленок, синтезированных в данной работе. Разложение проводилось на минимальное количество составляющих гауссовых пиков, при которых их результирующая кривая описывала экспериментальную кривую с достоверностью $>0.99 \%[11]$.

Перед разложением была проведена операция вычитания фона. Экспериментальные кривые представлены черной линией, гауссовы пики распределения обозначены зеленой линией, результирующая разложения показана красной линией. Из рис. 3 видно, что спектры RS раскладывались на 2 и на 3 пика в соответствии с максимальным значением достоверности.

В работах $[15,19,20]$ приведены спектры фононной плотности состояний (VDOS) графита и алмаза. Ocновные полосы VDOS алмаза находятся в диапазоне частот $1000-1335 \mathrm{~cm}^{-1}$, у графита - в диапазоне $1350-1600 \mathrm{~cm}^{-1}$.

Согласно работам [1,14-16], пик 3 в диапазоне частот $1500-1600 \mathrm{~cm}^{-1}$ можно отнести к $G$-пику (рис. 3). Пик 2 в области частот $1350-1400 \mathrm{~cm}^{-1}$ соответствует положению $D$-пика. Разложение рамановских спектров а-C пленок, синтезированных при температурах 50 и $150^{\circ} \mathrm{C}$, показывает, что на спектрах проявляется пик в области частоты $1260 \mathrm{~cm}^{-1}$ (пик 1 ), что соответствует фононной плотности состояний алмаза [15]. Таким образом, можно предположить, что синтезируемые аморфные углеродные пленки при температурах подложки менее $150^{\circ} \mathrm{C}$ структурированы алмазоподобными наночастицами. На 

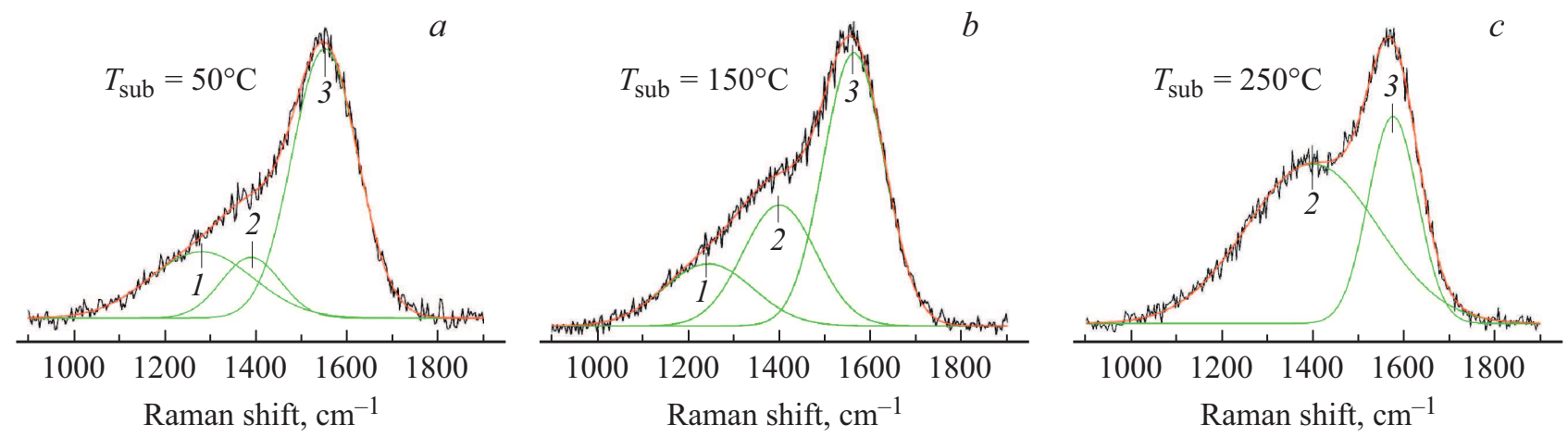

Рис. 3. Спектры КРС аморфных углеродных пленок, синтезированных при $p=1.2 \mathrm{~Pa}$ и $P_{\mathrm{d}}=2.1 \mathrm{~W} / \mathrm{cm}^{2}$ и разных температурах синтеза с применением разложения по методу Гаусса.

рис. 3, с пик 1 отсутствует, что согласуется с результатами работы [18], где показано резкое уменьшение содержания $s p^{3}$-узлов при $T_{\text {sub }}>200^{\circ} \mathrm{C}$.

Важно отметить, что в работе [18] а-С пленки синтезировались методом FCVA при фиксированной энергии конденсации $90 \mathrm{eV}$ в широком интервале температур подложки (от 100 до $200^{\circ} \mathrm{C}$ ) и содержали до $85 \% s p^{3}$-связей. Магнетронный ионно-плазменный метод синтеза, используемый в нашей работе, относится к неравновесным условиям для формирования структуры, т.е. такие условия синтеза характеризуются большим разбросом конденсируемых атомов, молекул и ионов углерода по энергии. Конденсируемые частицы с различной энергией создают условия для формирования структуры, которые существенно отличаются от условий в методе FCVA. Именно поэтому появление пика 1 в наших пленках существенно зависит от температуры подложки. Кроме этого, как отмечено в работе [21], алмазоподобные зерна с размерами $\sim 10 \AA$ и менее могут иметь более „мягкие объемного алмаза, что приводит к появлению VDOS в области $1260 \mathrm{~cm}^{-1}$. Как видно, на рис. 3 пик 1 смещается в низкочастотную область к полосе $1230 \mathrm{~cm}^{-1}$ с увеличением температуры синтеза $\left(T_{\mathrm{sub}}=150^{\circ} \mathrm{C}\right)$ и полностью исчезает при $T_{\mathrm{sub}}=250^{\circ} \mathrm{C}$. Такое поведение пика 1 показывает, что концентрация $s p^{3}$-узлов в аморфной углеродной пленке значительно уменьшилась. В этом случае приоритет в формировании структуры пленок переходит к $s p^{2}$-гибридизированным связям.

Как видно из рис. 3, интенсивность и полуширина пика 2 возрастают с увеличением температуры синтеза. Рамановские спектры а-C-пленок при $T_{\mathrm{sub}}=250^{\circ} \mathrm{C}$ раскладываются только на 2 составляющие - пик 2 и пик 3. Таким образом, как определили выше, структура описывается только $D$ - и $G$-пиками, т.е. вибрационными модами шестигранных молекул и $s p^{2}-\mathrm{C}-\mathrm{C}$-связями. Подобная картина изменения структуры, выявленная разложением спектров на гауссовы составляющие, наблюдается для всех режимов синтеза а-C в данной работе.
Размер структурных единиц, формирующих матрицу углеродной пленки, оценивают согласно правилу Туинстра и Коинга:

$$
I_{d} / I_{G} \propto 1 / L_{a},
$$

где $L_{a}$ - размер нанокластера. Однако в сильно неупорядоченных структурах дальнейшее увеличение беспорядка в атомной структуре приводит к уменьшению числа шестигранных молекул, что, в свою очередь, приводит к уменьшению интенсивности $D$-пика [15]. Поэтому отношение интенсивностей $D$ - и $G$-пиков $I_{D} / I_{G}$ начинает уменьшается с увеличением степени разупорядоченности структуры, и в этом случае уравнение Туинстра и Коинга неприменимо [15,22].

Появление колец в аморфной матрице указывает на возникновение определенной упорядоченности в аморфной структуре, что приводит к увеличению интенсивности $D$-пика. В этом случае $I_{D} / I_{G}$ пропорционально числу колец в кластере. Когда размер нанокластера, состоящий из шестигранных колец, не превышает $20 \AA$, необходимо использовать выражение

$$
\frac{I_{D}}{I_{G}}=c^{\prime}(\lambda) \times L_{a}^{2}
$$

При этом правильно использовать отношение не ширины на полувысоте пиков, а отношение их интенсивностей $I_{D} / I_{G}[11,22]$. Это объясняется тем, что интенсивность излучения рамановских спектров определяется фононными модами молекул, участвующих в наиболее вероятных резонансных процессах комбинационного рассеяния света. Подобные рассуждения можно провести и для кластеров пространственно-структурированных из $s p^{3}$-узлов. В [21] авторы обсудили интенсивность колебательных мод и их зависимость от волнового вектора и правил отбора. В данной работе утверждалось, что „запрет“ фонона допускает участие фононов с волновым вектором $q=2 \pi / d$, где $d$ - размер кластера, а не отдельных $s p^{2}$ - или $s p^{3}$-структурных единиц.

Расчет коэффициента пропорциональности в уравнении (2), согласно работе [15], для лазера с длиной волны 473 нт примерно равен $C^{\prime}(473) \approx 0.00376$. Таким 
образом, проведенная оценка в соответствии с соотношением $I_{D} / I_{G}$ показала, что размер графитоподобных кластеров увеличивается от $\sim 6 \AA$ до $\sim 12 \AA$ с повышением температуры.

Для алмазоподобной фазы, как показано и в работе [21], существование пика 1 обусловлено более мягкими кластерами из $s p^{3}$-узлов, размеры которых составляют $\sim 10 \AA$ и меньше. Кроме того, как следует из рис. 3, увеличение температуры синтеза приводит к уменьшению размеров этих кластеров. При температуре синтеза $250^{\circ} \mathrm{C} s p^{3}$-узлы не организуются в кластеры, а формируются как связывающие мостики между $s p^{2}$-структурно упорядоченными областями. В этом случае неупругое рассеяние света происходит на области, структурированной из $s p^{2}$-узлов, поэтому фононный спектр $s p^{3}$-узлов подавлен, и пик 1 исчезает.

Разложение на составляющие рамановских спектров a-C пленок, синтезированных при других значениях давления аргона и удельной мощности DC-разряда при температуре подложки $50^{\circ} \mathrm{C}$, примерно одинаковое и похоже на разложение, показанное на рис. $3, a$. Оценка размеров кластеров из $s p^{2}$-узлов выявила, что они имеют общую тенденцию к увеличению в интервале от 6 до $10 \AA$ с ростом удельной мощности. В то же время уменьшаются с повышением давления газа в том же диапазоне изменения $L_{a}$ при $T_{\mathrm{sub}}=50^{\circ} \mathrm{C}$. Обратная картина наблюдается с кластерами из $s p^{3}$-узлов.

Таким образом, применение нормального распределения при разложении рамановских спектров позволяет выявить зависимость изменения формируемой структуры а-С от условий конденсации и получить дополнительную информацию о размерах структурных единиц.

Нужно дополнительно отметить, как установлено авторами [23], что энергия сублимации алмазоподобных фаз меньше, чем энергия сублимации алмаза, что говорит об их меньшей термодинамической устойчивости. Поэтому такие фазы не могут быть получены в равновесных термодинамических условиях [23].

\section{Оптическая спектроскопия}

Как определили выше, термодинамические параметры существенно влияют на формирование атомной структуры аморфной углеродной пленки. Это влияние сказывается на соотношении $s p^{2} / s p^{3}$-связей и соответственно на размерах областей с квазиупорядоченной структурой. Оценка упорядоченности структуры показала, что при температурах менее $150^{\circ} \mathrm{C}$ в аморфной матрице присутствуют области из $s p^{3}$-узлов. Но с увеличением температуры синтеза наблюдается рост графитоподобных структур из $s p^{2}$-узлов.

В работе [17] наиболее подробно рассмотрен вопрос зонной структуры аморфного углерода. Было показано, что ширина запрещенной зоны обратно пропорциональна размерам нанокластеров из $s p^{2}$-узлов. Согласно модели неупорядоченных графитовых слоев с размерами около $15 \AA$ в диаметре, ограниченных $s p^{3}$-узлами, оптическая ширина запрещенной зоны соответствует $\sim 0.5 \mathrm{eV}$. Авторы [17] определили, что в основе формирования зонной структуры лежат $\pi$-взаимодействия между $\pi$-состояниями ближайших соседей первой и второй координационной сфер, которые составляют ближний порядок атомной структуры. Поэтому учли вклад каждого из $p \pi$ - и $p \sigma$-состояний в формирование краев зоны. В зависимости от процентного соотношения $s p^{2} / s p^{3}$-связей изменяется долевое участие $\pi$ и $\sigma$-электронов в формировании краев зоны и электронной плотности состояний (EDOS) вблизи уровня Ферми $\left(E_{\mathrm{F}}\right)$. При этом потолок валентной зоны, дно зоны проводимости и $\mathrm{EDOS}$ как внутри запрещенной зоны, так и вблизи $E_{\mathrm{F}}$ будут формироваться низкоэнергетическими электронами $\pi$ - $\pi^{*}$-состояний. Как было отмечено авторами [15,17], величина энергетического зазора между краями полос $\sigma-\sigma^{*}$ порядка $6 \mathrm{eV}$, поэтому уровни $\sigma$-состояний лежат далеко от уровня Ферми. Если в аморфной пленке концентрация $s p^{3}$-узлов более $20 \%$, то уменьшение доли $\pi$-электронов существенно влияет на EDOS вблизи уровня Ферми и на границах зоны $[17,18]$. Именно по этой причине $E_{g}$ увеличивается. В работаx $[17,18]$ показано, что количество $s p^{2}$-узлов и их ориентация существенно влияют на оптическую ширину запрещенной зоны.

Оптическая ширина запрещенной зоны является структурно чувствительным параметром, который дает существенный вклад в понимание зоной структуры и в ее формирование. Из спектров пропускания и отражения было определено, что зависимость коэффициента поглощения $(\alpha)$ от длины волны света в диапазоне значений $\alpha \sim 10^{3}-10^{5} \mathrm{~cm}^{-1}$ подчиняется квадратичному закону $\alpha h v=B\left(h v-E_{g}\right)^{2}$ Тауца $[18,24]$ для разрешенных прямозонных переходов. Ширина запрешенной зоны определялась в области значений $\alpha \sim 10^{5} \mathrm{~cm}^{-1}$ и $\alpha d \sim 1$. Зависимость $E_{g}$ от температуры подложки и давления газа $\mathrm{Ar}$ приведены на рис. 4. Как видно из рис. 4, $a$, изменение $E_{g}$ существенно зависит от температуры, и при $\Delta T_{\text {sub }}=200^{\circ} \mathrm{C}$ изменение $\Delta E_{g} \sim 1 \mathrm{eV}$. Подобная картина изменения зависимости $E_{g}\left(T_{\mathrm{sub}}\right)$ наблюдается и при других давлениях синтеза и $P_{\mathrm{d}}$.

Из рис. 4, $a$ видно, что увеличение температуры синтеза приводит к уменьшению $E_{g}$. Как было установлено выше, с увеличением температуры синтеза размеры графитоподобных нанокластеров из $s p^{2}$-узлов увеличиваются от 6 до $12 \AA \AA$. То есть наблюдаем увеличение области с квазиупорядоченной структурой, размеры которой можно отнести к среднему порядку атомной структуры. Изменение размеров нанокластеров из $s p^{2}$-узлов коррелирует с изменением величины $E_{g}$.

Как показано выше (рис. 2), при $T_{\text {sub }}=50^{\circ} \mathrm{C}$ положение $G$-пика постоянно для трех значений $P_{\mathrm{d}}$. И это постоянство положения $G$-пика говорит об одинаковом ближнем порядке атомной структуры. На рис. 4, $b$ при данных значениях синтеза пленок а-С мы наблюдаем зависимость $E_{g}$ как от удельной мощности DC-разряда, так 
$a$

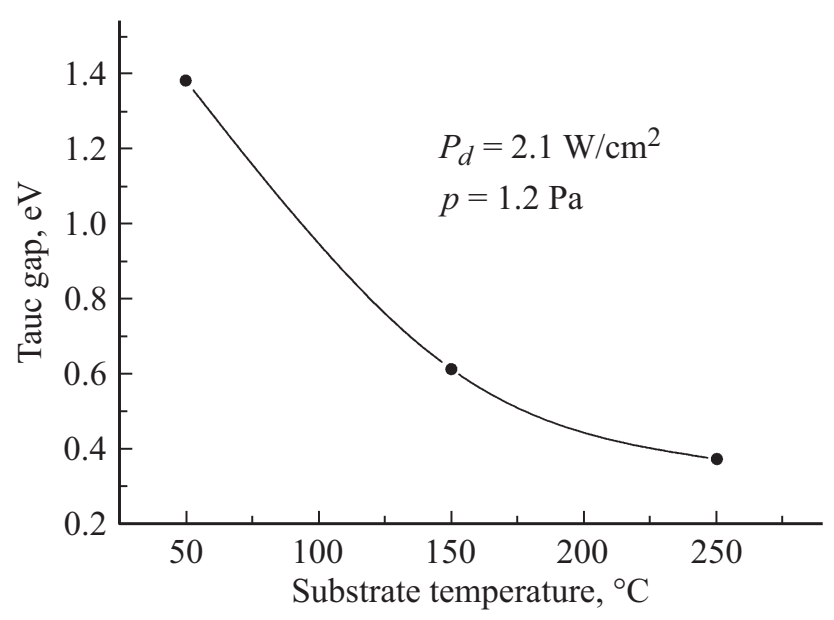

$b$

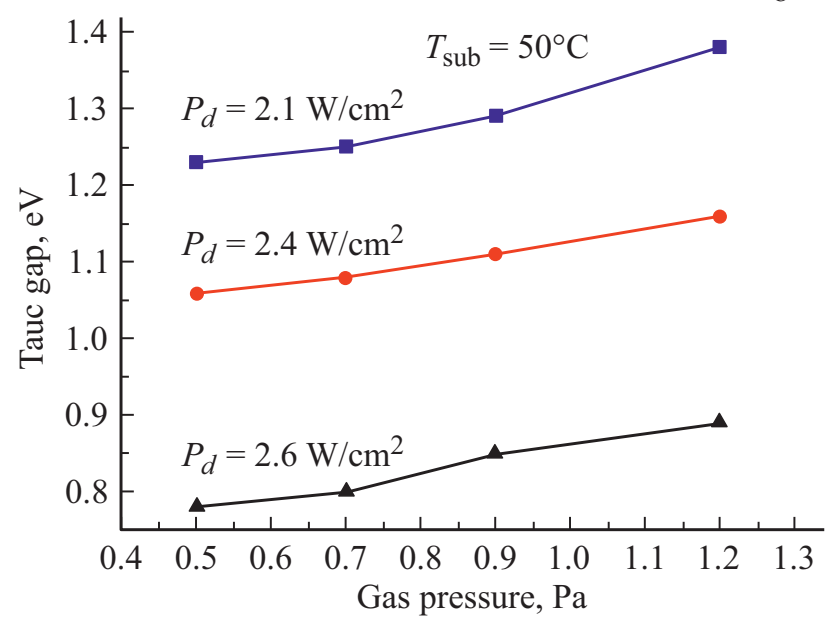

Pис. 4. Зависимость оптической ширины запрещенной зоны от $(a)$ температуры синтеза DLC-пленок и $(b)$ давления аргона для трех значений $P_{\mathrm{d}}$.

и от давления газа $\mathrm{Ar}$. С увеличением $P_{\mathrm{d}}$ на $0.5 \mathrm{~W} / \mathrm{cm}^{2}$ $E_{g}$ уменьшается в среднем на $\sim 0.47 \mathrm{eV}$. Помимо этого видно, что изменение $E_{g}$ прямо пропорционально изменению давления газа, т.е. при $P_{\mathrm{d}}=2.1 \mathrm{~W} / \mathrm{cm}^{2} E_{g}$ увеличивается на $0.15 \mathrm{eV}$ с увеличением $p$, и при $P_{\mathrm{d}}=2.6 \mathrm{~W} / \mathrm{cm}^{2} \quad \Delta E_{g}=0.1 \mathrm{eV}$. Такое изменение зоны говорит о существенном влиянии удельной мощности ионно-плазменного разряда и давления газа на формирование структуры. Как было определено выше, при $T_{\text {sub }}=50^{\circ} \mathrm{C}$ кластеры из $s p^{2}$ - и $s p^{3}$-узлов, формирующие a-C-пленку, имеют размеры больше радиуса второй координационной сферы. Размеры кластеров достигают $10 \AA$ и, как показывают расчеты, изменение среднего размера областей с $s p^{2}$-узлами примерно на $1 \AA$ приводит к изменению оптической ширины запрещенной зоны. Таким образом, это подтверждает предположение [17], что за формирование зонной структуры и распределение электронной плотности в зонах отвечает как ближний порядок, так и средний порядок атомной структуры в аморфном углероде, что возможно уникально и присуще только для а-С углерода.

Авторы работ $[1,15]$ утверждают, что если энергия запрещенной зоны $\mathrm{a}-\mathrm{C}$ пленок принимает значения в интервале от 0.2 до $1 \mathrm{eV}$, то такие пленки относят к графитоподобному аморфному углероду а-С. Если значения $E_{g}$ в диапазоне от 1 до $2.5 \mathrm{eV}$, то такие пленки по структуре относятся к тетрагональным аморфным углеродным пленкам ta-C, или их еще называют алмазоподобными $[1,14]$.

\section{XPS-спектроскопия}

Для изучения соотношения гибридизаций $s p^{2} / s p^{3}$-связей на поверхности пленок провели исследования методом XPS. На рис. 5 приведен общий спектр XPS, характерный для всех исследуемых

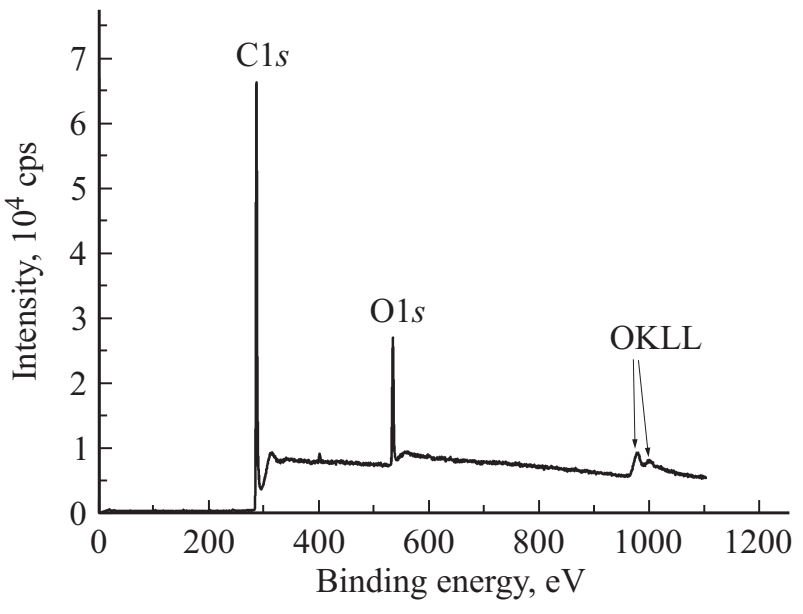

Рис. 5. XPS а-С-пленки, синтезированной при $2.1 \mathrm{~W} / \mathrm{cm}^{2}$.

образцов, откуда видно, что в углеродной пленке присутствует определенное количество кислорода $(\leq 10$ at.\%) и гораздо в меньшей степени азота (< 1.3 at.\%). Появление кислорода и азота в а-С-пленке связано с адсорбцией из атмосферы. Углеродный пик C1s лежит в области с энергией $\sim 285.2 \mathrm{eV}$ и характерен для всех образцов, полученных при температуре меньше $50^{\circ} \mathrm{C}$.

Для определения концентрации $s p^{3}$-связей провели разложение C1s-пика на составляющие (рис. 6). Фон вычитался методом Ширли. Положение пика в области $284.9 \mathrm{eV}$ соответствует $s p^{2}$-C-C-связям, в области $285.25 \mathrm{eV}$ соответствует $s p^{3}$-C-C-связям, максимумы гауссовых пиков, лежащие в области более $286 \mathrm{eV}$, соответствуют связям $\mathrm{C}-\mathrm{O}$ и $\mathrm{C}-\mathrm{N}$. Как видно, доля $s p^{3}$-связей с увеличением удельной мощности уменьшается, при этом доля $s p^{2}$-связей увеличивается. Оценку концентрации $s p^{2}$ - и $s p^{3}$-С-С-связей проводили по площадям гауссовых пиков с учетом влияния связей 

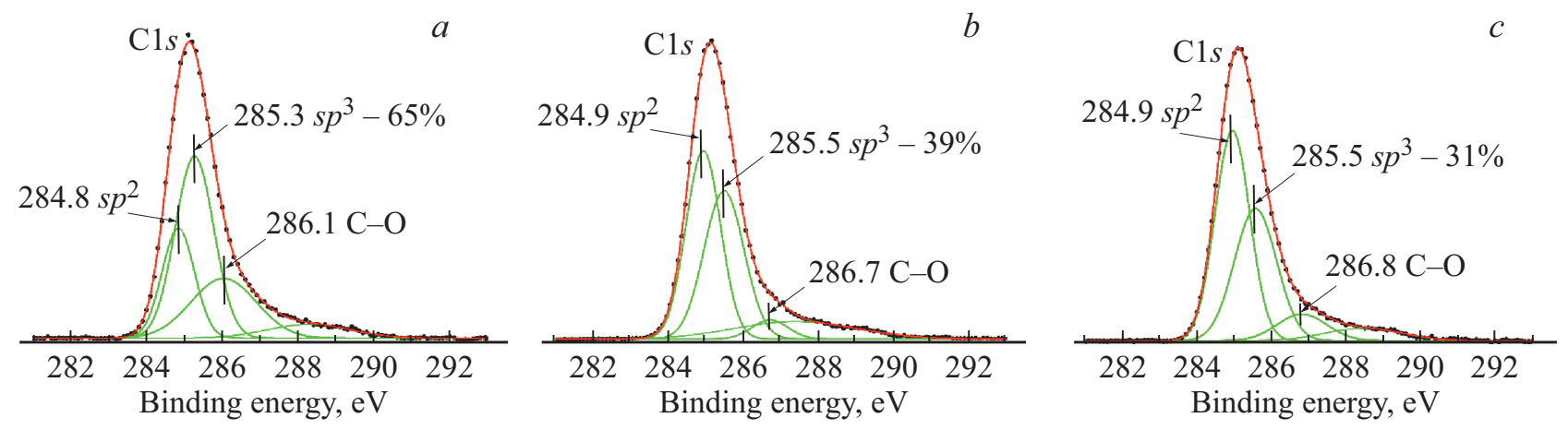

Рис. 6. XPS а-С-пленок, синтезированных при $T_{\text {sub }}=50^{\circ} \mathrm{C}$ и разной удельной мощности: $(a) 2.1 \mathrm{~W} / \mathrm{cm}^{2},(b) 2.4 \mathrm{~W} / \mathrm{cm}^{2}$, (c) $2,6 \mathrm{~W} / \mathrm{cm}^{2}$.

$\mathrm{C}-\mathrm{O}$ и $\mathrm{C}-\mathrm{N}$. Процентное содержание $s p^{3}$-связей для каждой мощности синтеза приведены на рис. 6. Увеличение концентрации $s p^{2}$-связей с увеличением удельной мощности разряда соответственно равно 30,50 и $53 \%$. Сопоставляя результаты, приведенные на рис. $4, b$ и 6 , можно видеть определенную корреляцию в изменении оптической ширины запрещенной зоны и соотношения $s p^{2} / s p^{3}$-связей.

\section{Заключение}

Использование электромагнита в магнетроне позволяет поддерживать отношение $E / p$ постоянным в процессе синтеза. Напряжение и ток плазмы DC-разряда корректировались величиной магнитного поля магнетрона, что позволило детально провести исследование влияния термодинамических и кинетических параметров синтеза на структуру и свойства наногетероморфных углеродных пленок. При этом появилась возможность при одинаковом значении тока DC-разряда провести исследование влияния давления газа для трех различных удельных мощностей плазменного разряда на формирование структуры, а так же выявить влияние структуры на оптическую ширину запрещенной зоны. Методами рамановской, XPS и оптической спектроскопии показано существование определенных закономерностей между условиями синтеза, структурой и оптической запрещенной зоной в аморфных углеродных пленках.

Углеродные пленки, синтезированные в неравновесных условиях, имеют неупорядоченную структуру, состоящую из графитоподобных и алмазоподобных нанокластеров. Размеры этих нанокластеров зависят от термодинамических и кинетических параметров синтеза и варьируются от 6 до $12 \AA$. Размеры кристаллоподобных частиц соизмеримы со средним порядком структуры, и, как показано в данной работе, изменение размеров областей среднего порядка приводит к изменению ширины запрещенной зоны.

Оптическая ширина запрещенной зоны принимает значение меньше $1 \mathrm{eV}$ в а-C-пленках, синтезированных при $50^{\circ} \mathrm{C}$ и удельной мощности плазменного разряда $2.6 \mathrm{~W} / \mathrm{cm}^{2}$. Такие углеродные пленки в большей степени состоят из графитоподобной структуры. При этом положение $G$-пика определяется при $1545 \mathrm{~cm}^{-1}$, что указывает на формирование структур из $s p^{3}$-узлов, т.е. алмазоподобной структуры. Однако общепринято, что к алмазоподобным пленкам нужно относить а-С-пленки, значения $E_{g}$ которых больше $1 \mathrm{eV}$. Таким образом, видим неоднозначность в характеризации атомной структуры при температуре синтеза $50^{\circ} \mathrm{C}$.

Из сказанного можно заключить, что изучение структуры аморфных углеродных пленок RS существенно дополняется оптическими методами исследования и рентгеновской фотоэлектронной спектроскопией. Параметр $E_{g}$ определяется распределением EDOS краев разрешенных зон, которая формируется относительной концентрацией $s p^{2}$ - и $s p^{3}$-узлов, что было подтверждено данными XPS-анализа.

\section{Финансирование работы}

Работа выполнена в рамках грантового финансирования № АР05131495 комитета науки МОН Республики Казахстан.

\section{Конфликт интересов}

Авторы заявляют, что у них нет конфликта интересов.

\section{Список литературы}

[1] Robertson J. // Mater. Sci. Eng. 2002. R37. P. 129.

[2] Juan Manuel Mendez, Stephen Muhl, Contreras-Puente G., Aguilar-Hernandez J. // Thin Solid Films. 1992. V. 220. P. 125.

[3] Equer B., Drevillon B., French I., Kallfass T. Thin Film Materials for Large Area Electronics. Elsevier Science. 1999. V. 80. 1st Edition. Hardcover, 978-0-08-043607-4

[4] Grierson D.S., Carpick R.W. // Nano Today. 2007. V. 2. N 5. P. 12.

[5] Nilgün Doğan Baydoğan // Materials Science and Engineering. 2004. B. 107. P. 70. 
[6] Wang H., Guo J.Q., Zhou Y.S. // Carbon. 2013. V. 64. P. 67.

[7] Hevia S.A., Bejide M., Duran B., Rosenkranz A., Ruiz H.M., Favre M., del Rio R. // J. Solid State Electrochemistry. 2018. V. 22. N 9. P. 2845.

[8] Gangopadhyay A. // Tribology Letters. 1998. V. 5. N 1. P. 25.

[9] Shum P.W., Zhou Z.F., Li K.Y., Chan C.Y. // Thin Solid Films. 2004. V. 458. P. 203.

[10] Yongjun Wanga, Hongxuan Lia, Li Jia, Fei Zhaoc, Qinghua Konga, Yongxia Wanga, Xiaohong Liua, Weilong Quana, Huidi Zhoua, Jianmin Chena // Surface and Coatings Technology. 2011. V. 205. N 8-9. P. 3058.

[11] Ryaguzov A.P., Yermekov G.A., Nemkayeva R.R., Guseinov N.R., Aliaskarov R.K. // J. Mater. Res. 2016. V. 31. N 1. P. 127.

[12] Ferrari A.C., Robertson J. // Phil. Trans. R. Soc. Lond. A. 2004. V. 362. P. 2477.

[13] Casiraghi C., Piazza F., Ferrari A.C., Grambole D., Robertson J. // Diamond \& Related Materials. 2005. V. 14. P. 1098.

[14] Ferrari A.C. // Diamond and Related Materials. 2002. V. 11. P. 1053.

[15] Ferrari A.C., Robertson J. // Phys. Rev. B. 2000. V. 61 (20). P. 14095.

[16] Ferrari A.C., Robertson J. // Phys. Rev. B. 2001. V. 64. P. 075414.

[17] Robertson J., O’Reilly E.P. // Phys. Rev. B. 1987. V. 35. N 6. P. 2946.

[18] Chhowalla M., Robertson J., Chen C.W., Silva S.R.P., Davis C.A., Amaratunga G.A.J., Milne W.I. // J. Appl. Phys. 1997. V. 81. P. 139.

[19] Knight D.S., White W.B. // J. Mater. Res. Society. 1989. V. 4. N 2. P. 385.

[20] Tuinstra F., Koening J.L. // J. Chem. Phys. 1970. V. 53. P. 1126.

[21] Ferrari A.C., Robertson J. // Phys. Rev. B. 2001. V. 63. P. $121405 \mathrm{R}$.

[22] Cancado L.G., Jorio A., Martins Ferreira E.H., Stavale F., Achete C.A., Capaz R.B., Moutinho M.V.O., Lombardo A., Kulmala T.S., Ferrari A.C. // Nano Lett. 2011. V. 11. P. 3190.

[23] Беленков Е.А., Грешняков В.А. // ФТТ. 2013. Т. 55. № 8. C. 1640; (Belenkov E.A., Greshnyakov V.A. // Physics of the Solid State. 2013. V. 55. N 8. P. 1754.)

[24] Tauc J. // Prog. Semicond. 1965. V. 9. P. 89. 